Proceedings

\title{
Eccentric Computational Embodiments: Cognitive Domestication of External Entities ${ }^{\dagger}$
}

\author{
Lorenzo Magnani \\ Department of Philosophy and Computational Philosophy Laboratory, University of Pavia, 27100 Pavia, \\ Italy; lmagnani@unipv.it \\ + Fourth International Conference on Philosophy of Information, Berkeley, California, USA, 2-6 June 2019.
}

Published: 15 May 2020

\begin{abstract}
Eco-cognitive computationalism sees computation in context, adopting the intellectual visions advanced by the cognitive science perspectives on embodied, situated, and distributed cognition. It is in this framework that we can fruitfully study the relevance in recent computer science devoted to the simplification of cognitive and motor tasks generated in organic entities by the morphological aspects. Ignorant bodies can be cognitively "domesticated" to become useful "mimetic bodies", which originate eccentric new computational embodiments capable of rendering an involved computation simpler and more efficient. On the basis of these considerations, we will also see how the concept of computation changes, being related to historical and contextual factors, so that the "emergence" of new kinds of computations can be epistemologically clarified, such as the one regarding morphological computation. Finally, my presentation will introduce and discuss the concept of overcomputationalism, as intertwined with the traditional concepts of pancognitivism, paniformationalism, and pancomputationalism, seeing them in a more naturalized intellectual disposition, more appropriate to the aim of bypass ontological or metaphysical overstatements.
\end{abstract}

Keywords: morphological computation; physical computation; mimetic bodies; computational substrates; pancomputationalism; simplexity

\section{Building Computational Mimetic Bodies through Morphology-Based Enhancing}

The recent emphasis on the simplification of cognitive and motor tasks generated in organic agents by morphological aspects implies - once exploited in robotics - the construction of appropriate mimetic bodies able to render an accompanied and integrated computation simpler, according to a general appeal to the simplexity of animal embodied cognition, which stresses possible complementary relationships between complexity and simplicity. To comprehend this process, it is first of all necessary to illustrate various aspects of the so-called physical computation.

\section{A Computer Is a Physical System}

In my book [1], I have illustrated some aspects of the relationships between the concepts of information, cognition, and computation, trying to ask the question "Is cognition computation?" We can give a positive answer, it is, but it is not only computation, so that we cannot identify cognition with computation. Of course, information processing and computation are involved in cognition, and plenty of research has been done to clarify the various roles and types of computation and information processing implicated in cognition, even if these kinds of research, at least from an eco-cognitive perspective, are damned to become old-fashioned. I prefer to affirm, from a perspective informed by a kind of naturalistic epistemology, that, given the fact the concept of computation changes and is subject to meaning variations, the other two concepts, already individuated in themselves by 
precarious definitions, being associated with the developments of knowledge, technologies, and cultural frameworks, also vary. Recent rich and informed studies fruitfully aim at disambiguating the concept of digital computation in contemporary cognitive science, by illustrating how digital computation is implemented in physical systems: these studies do not end up in pancomputationalism, that is, the view that every physical system is a digital computing one and can be described in computational terms.

These studies mainly aim at philosophical/ontological and definitory results. My concern here is more humble: my eco-cognitive computationalism does not aim at furnishing an ultimate and static definition of the concepts of information, cognition, and computation, such as a textbook or a more "analytic" perspective can provide. Instead, by valuing their historical and dynamical aspects, I will suggest an epistemological perspective that depicts how we can understand the change and extension of their meanings thanks to the example (and the description of the "emergence") of what I call the new computational "domestication" of physical entities, thanks to morphological computing. To this aim, I will adopt the perspective on physical computation offered by ([2], p. 14), that is particularly appropriate to favor my concerns.

1. A computer is a physical system with actual constituent parts and its own internal interactions that take it from one physical state to another.

2. Hence, I agree with Horsman et al., who contend that physical computing is "the use of a physical system to predict the outcome of an abstract evolution" ([2] p. 14). Indeed, it is interesting to note that, in computations, we do not have to deal with a physical system that needs to be described (this is, for example, the case of physics), but, on the contrary, with an abstract object that we want to evolve, thanks to the physical system itself. Once we have realized this exploitation of a physical entity, the physical evolution performs a computation, so is interpreted by a human or another artificial agent.

3. A computer is usually a technology built by using our scientific theories to precision-engineer physical systems to desired specifications.

4. A physical system is computing when a special relationship of abstract mathematical/logical entities to the physical ones at stake is enforced.

5. Beyond conventional and unconventional cases, the notion of computation, and its related system property, information, have been imported into other fields in an attempt to describe and "explain" such diverse processes as photosynthesis and the conscious mind, and a strand of modern cross-disciplines have given us the claims that "everything is information" or "the universe is a quantum-computer" or "everything computes" ([2], p. 2). Obviously, many researchers plausibly contend that, defining the universe and everything in it as a computer, the notion of physical computation becomes empty. I will come back to this issue below in Section 3 .

\section{Morphological Computation}

I have also illustrated the recent results of morphological computation [3], in which the emphasis on the simplification of cognitive and motor tasks has rendered possible the construction of appropriate "mimetic bodies" able to render accompanied computations simpler, according to a general appeal to the "simplexity" of animal embodied cognition. I have stressed that, in the case of morphological computation, we can surely conclude that we are facing a new activity of what we can call "distributed computation": the promise of morphological computation principles in robot design can originate a new generation of robots with better adaptability and restricted number of required control parameters. Finally, I have also dedicated a short discussion to the concepts of paninformationalism and pancomputationalism, showing that the framework of distributed computation helps us see them in a more naturalized and prudent perspective, avoiding ontological or metaphysical considerations. I also devoted a part of the final considerations to illustrating the related problems regarding the epistemological limitations of computational modeling, when used to simulate the behavior of a physical or a biological system. 
Funding: This research was funded by Funds: Blue Sky Research 2017 (Grant No. BSR1780130) - University of Pavia, Pavia, Italy.

Conflicts of Interest: The authors declare no conflict of interest.

\section{References}

1. Magnani, L. The Abductive Structure of Scientific Creativity; Springer: Cham, Switzerland, 2017.

2. Horsman, C.; Stepney, S.; Wagner, R. C.; Kendon, V. When does a physical system compute? Proc. R. Soc. A 2014, 470, 1-25, doi:10.1098/rspa.2014.0182.

3. Müller, V.C.; Hoffmann, M. What is morphological computation? On how the body contributes to cognition and control. Artif. Life 2017, 23, 1-24.

(C) 2020 by the authors. Licensee MDPI, Basel, Switzerland. This article is an open access article distributed under the terms and conditions of the Creative Commons Attribution (CC BY) license (http://creativecommons.org/licenses/by/4.0/). 